\title{
Ultrahigh sensitivity of slow-light gyroscope
}

\author{
U. Leonhardt ${ }^{1,2}$ and P. Piwnicki ${ }^{2}$ \\ ${ }^{1}$ School of Physics and Astronomy, University of St Andrews, North Haugh, St Andrews, Fife, KY16 9SS, Scotland \\ ${ }^{2}$ Physics Department, Royal Institute of Technology (KTH), Lindstedtsvägen 24, S-10044 Stockholm, Sweden
}

Slow light generated by Electromagnetically Induced Transparency is extremely susceptible with respect to Doppler detuning. Consequently, slow-light gyroscopes should have ultrahigh sensitivity.

42.50.Gy

In recent experiments [1] light has been slowed down dramatically to just a few meters per second. Traveling at this incredibly low speed, light is sensitive enough to serve in detections of subtle motion effects such as the optical Aharonov-Bohm effect of quantum fluids [2]. A moving medium is able to drag light, and this dragging phenomenon gives rise to the sensitivity with respect to motion. Analyzed more carefully [2 5], a moving medium appears as an effective change of the space-time metric (as an effective gravitational field). When the medium outruns the light traveling inside, suitable flows may appear as optical black holes [2]. Apart from detecting quantum flows and from creating artificial black holes, is there a practical application for the incredible motion sensitivity of slow light?

Optical gyroscopes are regularly employed to sense motion. What would be the advantage of a slow-light gyroscope? How do passive dielectric gyroscopes work? Imagine for simplicity a solid block of uniform dielectric material that is rotating at angular velocity $\Omega_{0}$. (In a real fiber-gyroscope light travels in a multitude of coils, for enhancing the effect.) We assign cylindrical coordinates to the block where the $z$ direction coincides with the rotation axis. First, assume an essentially non-dispersive material such as glass that is characterized by the refractive index $n$. In this material, light experiences the space-time metric [3]

$$
d s^{2}=\frac{c^{2} d t^{2}}{n^{2}}+2 \alpha \Omega_{0} r d t d \varphi-d r^{2}-r^{2} d \varphi^{2}-d z^{2}
$$

in the limit of low rotation velocities $\Omega_{0} r$ compared with the speed of light in vacuum, $c$. Fresnel's dragging coefficient,

$$
\alpha=1-\frac{1}{n^{2}},
$$

quantifies the degree to which light is forced to move along with the medium. Clearly, $\alpha$ vanishes in the absence of a medium $(n=1)$ and $\alpha$ approaches unity in the limit of a very strong medium $(n \rightarrow \infty)$. Now, imagine that the block of dielectric material is at rest and instead of the medium the laboratory frame is rotating at angular velocity $\Omega$. We obtain from the space-time metric of light in the medium frame,

$$
d s^{2}=\frac{c^{2} d t^{2}}{n^{2}}-d r^{2}-r^{2} d \varphi^{\prime 2}-d z^{2}
$$

the metric in the rotating laboratory frame by the simple transformation

$$
\varphi^{\prime}=\varphi-\Omega t .
$$

To leading order in $\Omega r / c$ the transformed metric (3) coincides with the metric (11) of light in the rotating block if we put

$$
\Omega=\alpha \Omega_{0} .
$$

A rotating dielectric and a dielectric in a rotating frame are practically equivalent, yet they appear to rotate at different angular velocities. Fresnel's dragging coefficient (2) quantifies the ratio between the actual and the apparent angular velocity of the rotating dielectric body, $\Omega_{0}$ and $\Omega$, respectively. The coefficient $\alpha$ characterizes the degree to which light follows the actual rotation and, consequently, the degree to which rotation can be detected by optical interference.

Slow light [1] has been generated using Electromagnetically Induced Transparency (EIT) [6]. EIT takes advantage of a quantum-interference effect in multi-level atoms, bought about by dressing the atoms with the light of an appropriate auxiliary beam. A probe beam at resonance frequency can travel though the EIT medium that would be totally opaque without the assistance of the auxiliary light. Exactly on resonance a continuous probe wave travels at a phase velocity of $c$, i.e. the medium has a refractive index of unity. On the other hand, the EIT resonance is very sharp and is ultrasensitive with respect to frequency detuning. An EIT medium is thus extremely dispersive and, in turn, short light pulses having an extended spectrum travel at a very low group velocity $v_{g}$. Another aspect of the extreme dispersion of an EIT medium is the ultrasensitivity with respect to the Doppler detuning due to motion.

So, what happens if we replace the rotating block of non-dispersive material by an EIT medium of extreme dispersion? How large is the dragging coefficient? Let us determine the space-time line element,

$$
d s^{2}=g_{\mu \nu} d x^{\mu} d x^{\nu} \quad, \quad d x^{\mu}=(c d t, d \mathbf{x}),
$$

i.e. the covariant metric tensor $g_{\mu \nu}$. For this we invert the contravariant metric of slow light [2], $g^{\mu \nu}$, to lowest order in the ratio between the medium velocity and the velocity 
of light, $c$. In the limit of slow rotations, the line element turns out to have the same structure as the metric (11) of non-dispersive media with, however, a refractive index of unity and a modified dragging coefficient of

$$
\alpha=\frac{c}{v_{g}}-1 .
$$

Compared with passive dielectric gyroscopes, a slow light gyroscope operated with light of a modest group velocity of kilometers per second increases the rotation sensitivity by five orders of magnitude, and an ambitious group velocity of meters per second amounts to a fantastic improvement by eight orders of magnitude. Of course, for this one would need to create slow light in a solid block of material in order to attach the gyroscope to the rotating body one is interested in. Solid-state media tend to destroy the quantum-interference conditions of EIT much more rapidly than gases or Bose-Einstein condensates [1]. However, first demonstrations of EIT in solids have been already reported [7] and an interesting proposal of EIT in semiconducters has been recently published [8]. It would be desirable to demonstrate unambiguously slow light in solids, stimulated perhaps by the potential advantage of slow light gyroscopes: ultrahigh motion sensitivity.

\section{ACKNOWLEDGEMENTS}

We are grateful to Malcolm Dunn and Stig Stenholm for valuable discussions. U.L. gratefully acknowledges the support of the Alexander von Humboldt Foundation and of the Göran Gustafsson Stiftelse.

[1] L. V. Hau, S. E. Harris, Z. Dutton, and C. H. Behroozi, Nature 397, 594 (1999); M. M. Kash, V. A. Sautenkov, A. S. Zibrov, L. Hollberg, H. Welch, M. D. Lukin, Y. Rostovsev, E. S. Fry, and M. O. Scully, Phys. Rev. Lett. 82, 5229 (1999); D. Budiker, D. F. Kimball, S. M. Rochester, and V. V. Yashchuk, ibid. 83, 1767 (1999).

[2] U. Leonhardt and P. Piwnicki, Phys. Rev. Lett. 84, 822 (2000).

[3] U. Leonhardt and P. Piwnicki, Phys. Rev. A 60, 4301 (1999).

[4] W. Gordon, Ann. Phys. (Leipzig) 72, 421 (1923).

[5] Pham Mau Quan, C. R. Acad. Sci. (Paris) 242, 465 (1956); Archive for Rational Mechanics and Analysis 1, $54(1957 / 58)$.

[6] P. L. Knight, B. Stoicheff, and D. Walls (eds.), Phil. Trans. R. Soc. Lond. A 355, 2215 (1997); S. E. Harris, Phys. Today 50(7), 36 (1997); M. O. Scully and M. Zubairy, Quantum Optics (Cambridge University Press, Cambridge, 1997).

[7] Y. Zhao, C. Wu, B.-S. Ham, M. K. Kim, and E. Awad, Phys. Rev. Lett. 79, 641 (1997); B.-S. Ham, M. S. Shariar, and P. R. Hemmer, Opt. Lett. 22, 1138 (1997); K. Ichimura, K. Yamamoto, and N. Gemma, Phys. Rev. A 58, 4116 (1998).

[8] M. Artoni, G. C. La Rocca, and F. Bassani, Europhys. Lett. 49, 445 (2000) 